\title{
Molecular Dynamics Simulations of Nanoclusters for Improved STEM Image Simulations
}

\author{
Richard Aveyard*, Jun Yuan*, Ziyou Li** and Roy L. Johnston***. \\ *Department of Physics, University of York, Heslington, York, YO10 5DD, United Kingdom. \\ **Nanoscale Physics Research Laboratory, School of Physics and Astronomy, University of Birming- \\ ham, Edgbaston, Birmingham B15 2TT, United Kingdom. ***School of Chemistry, University of \\ Birmingham, Edgbaston, Birmingham B15 2TT, United Kingdom.
}

Gold nanoclusters are of particular interest to the catalysis industry as it has been found that, whilst inert in bulk form, gold is a highly effective catalyst for the oxidation of carbon monoxide in nanocluster form. Furthermore, there has been found to be a strong relationship between particle size and catalytic activity which is yet to be understood [1]. The structural characterization of nanoclusters is paramount to elucidating the fundamental causes of such size effects. The use of electron microscopy techniques in producing atomic resolution images in two-dimensions is now well established but extending their abilities to determining the three dimensional structure of specimens is more challenging. Electron tomography can be used to produce 3-dimensional images but the resolution of this technique is too low to determine the positions of individual atoms within nanoclusters and the requirement for multiple probe-sample interactions renders this technique unsuitable for more unstable structures. The first major development in extracting atomic-scale three-dimensional information from single 2-dimensional electron microscope images was reported by Young [2]. This work confirmed a linear relationship between the integrated intensity of images from high angle annular dark field scanning transmission electron microscopy (HAADF STEM) of nanoclusters and the number of atoms in the cluster. In $2008 \mathrm{Li}$ et. al. reported on the successful $3 \mathrm{~d}$ characterization of gold nanoclusters[3]. This was achieved by comparing the intensity profile of HAADF STEM experimental images with simulated images of predicted structures. A good match between experimental and simulated images was found, however, there were discrepancies. These incongruities were particularly pronounced at the cluster surface and were attributed to the thermal vibrations of atoms which were only accounted for in a simplistic manner due to a lack of more detailed information.

In this work the thermal motion of individual atoms in gold nanoclusters has been investigated using molecular dynamics simulations so that the effects of thermal diffuse scattering on HAADF STEM images may be quantified and included in computational image simulations. Molecular dynamics simulations have beenperformedona309 atom gold nanocluster with ino-decahedral geometry. This system was chosen as it is predicted by the shell stability model to be a stable structure with high experimental abundance and so should prove a convenient candidate for experimental comparison [4]. $\mathrm{Li}$ et. al. reported that at room temperature, $32 \%$ of nanoclusters with $309 \pm 6$ atoms are of inodecahedral structure[3]. Simulations were performed using the DLPOLY molecular dynamics package [5] with interatomic interactions modeled by an empirical Gupta potential [6]. The simulations were run for $0.5 \mathrm{~ns}$ including an equilibration period of $0.1 \mathrm{ps}$. The forces and trajectories were calculated every $2 \mathrm{fs}$. 
Simulations were carried out with a Hoover NVT ensemble. The square displacements of each atom from its origin (def ned by its average position) were calculated at each time step in order to calculate mean squaredisplacements (MSDs) over the length of the simulation.

Fig.(1) shows the mean-square displacement of atoms at varying unique positions within the cluster. As expected, the core atom exhibits the least thermal motion whilst the surface vertex atoms, those with the lowest co-ordination, show the largest vibration amplitudes. The MSD of atoms of different positions within the frst, second and third shells are largely very similar which suggests that a shell average will be suff cient to account for position dependent thermal scattering effects in image simulations. In the outer shell, however there is a large variation in the MSDs of atoms at different positions with the difference in MSD between the vertex and tip atoms being greater than the difference between the core atom and the tip atoms in the outer shell. This suggests that the dynamic effects on image simulations should ideally be determined for each unique atom position.

In summary, molecular dynamics simulations have been carried out to determine the position dependent atomic mean square displacement of atoms so that they can be used for realistic simulations of STEM images of atomic clusters.

\section{References}

[1]M Haruta, Journal of Catalysis, 144(1) (1993) 175.

[2]N Young et al., Physical Review Letters., 101(24) (2008) 28-31.

[3]ZY Li et al., Nature, 451(7174) (2008) 46.

[4]T Martin, Physics Reports, 273(4) (1996) 199-241.

[5]W Smith and TR Forester, The DL_POLY Molecular Simulation Package, URL: http: //www . dl . ac.uk/TCSC/Software/DL\_POLY

[6]F Cleri and V Rosato, Physical Review B., 48(1) (1993) 22-33.

[7]This work is supported by EPSRC grant EP/G070474/1 and G070326/1

a)

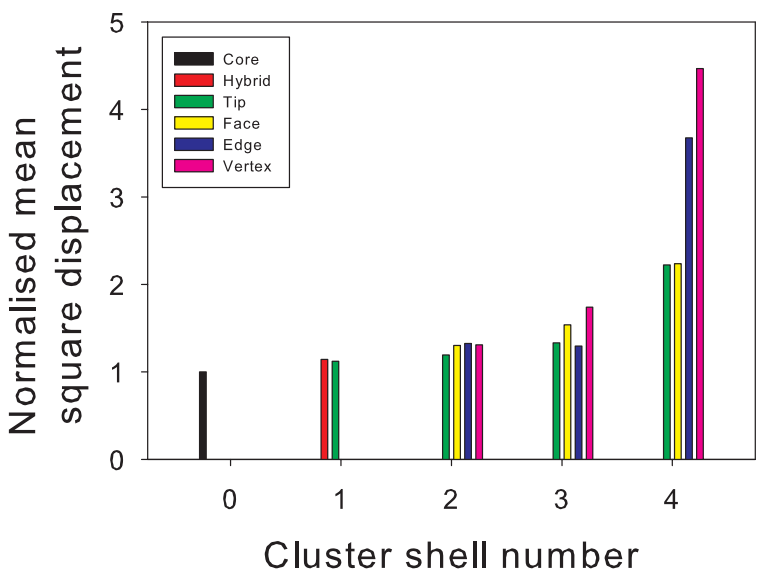

b)

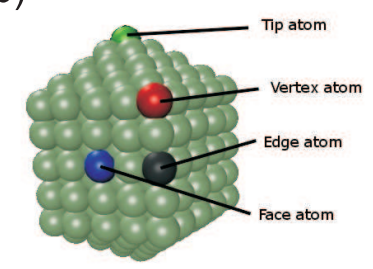

c)

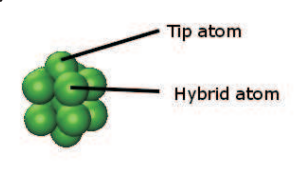

Figure 1: (a)Position dependence of the mean square displacement of atoms in a 309 ino-decahedral gold cluster at 300K. (b) Diagram indicating unique atom positions in the outer shell of a gold cluster. (c) Diagram highlighting hybrid face/edge/vertex atoms in the f rst cluster shell. 